\title{
Mehrwertdienst im Lebenszyklus von Sachanlagen in der Logistik
}

\author{
Prof. DR.-ING AXEL KUHN \\ DiPL.-ING. ANDREAS HELLMANN \\ DR.-ING. FRANK ELLERKMANN \\ FRAUNHOFER-INSTITUT FÜR MATERIALFLUSS UND LOGISTIK IML
}

Unter dem General-Thema „Hybride Wertschöpfungsketten“ sind Zukunftsaufgaben subsummiert, die eine maßgebliche Verbesserung der Kooperationsprozesse vieler unterschiedlicher Dienste aus einem Leistungsobjekt verfolgen. Wenn das Leistungsobjekt eine Anlage ist, dann sind Wertschöpfungsketten gemeint, die von einer ersten Nutzungsspezifikation bis zur Verschrottung reichen. Mit Wertschöpfung sind sowohl Leistungen zur Herstellung und Integration dieser Anlagen in Betreiberprozesse bezeichnet, als auch diejenigen, die einen Beitrag dieser Anlage zur Wertschöpfung beim Nutzer leisten. Mit Mehrwertdiensten sind somit alle Leistungen gemeint, die an der Anlage oder für die Anlage während ihres gesamten Anlagenlebenszyklus erbracht werden. Sie dienen zur Nutzenerhöhung beim Betreiber, dem Nutzer und dem Anlagenhersteller. Damit sind Mehrwertdienste in Hybriden Wertschöpfungsketten grundsätzlich kollaborative Dienste, die üblicherweise konfliktäre Ziele mehrerer (oder sogar vieler) Interessen auflösen müssen.

Under the general subject „Hybride value-added chains“ future projects are summarized which aim at improving the cooperation of different services of one service object. When this service object is a plant the value-added chains encompass the first specification of an application up to the scrapping at the end of its lifetime. Value-added chains include services for the production and integration of this plant into operating processes as well as services of value to the user. Value-added services are all those performances provided by the plant or for the plant during its complete lifetime and should increase the benefit for the operator, the user and the manufacturer. For this reason, in hybride value-added chains services always are collaborative and have to coordinate the conflicting aims of several (or even many) interests.

\section{Hybride Wertschöpfungsketten}

Aus dem Begriff Hybrid folgert man [Duden04]: „aus verschiedenem zusammengesetzt, von zweierlei Herkunft“; somit sollte die Bezeichnung „hybride Wertschöpfungskette“ zunächst einmal auf alle Wertschöpfungsketten angewendet werden, die sich in irgendeiner Form aus unterschiedlichen Elementinteressen zusammensetzen, die es zu koordinieren, kombinieren oder konstituieren gilt. Die „Neue Institutionenökonomie“ als moderne volkswirtschaftliche Richtung definiert drei Koordinationsformen des wirtschaftlichen Handelns: den Markt, die Hierarchie und die Hybride. 


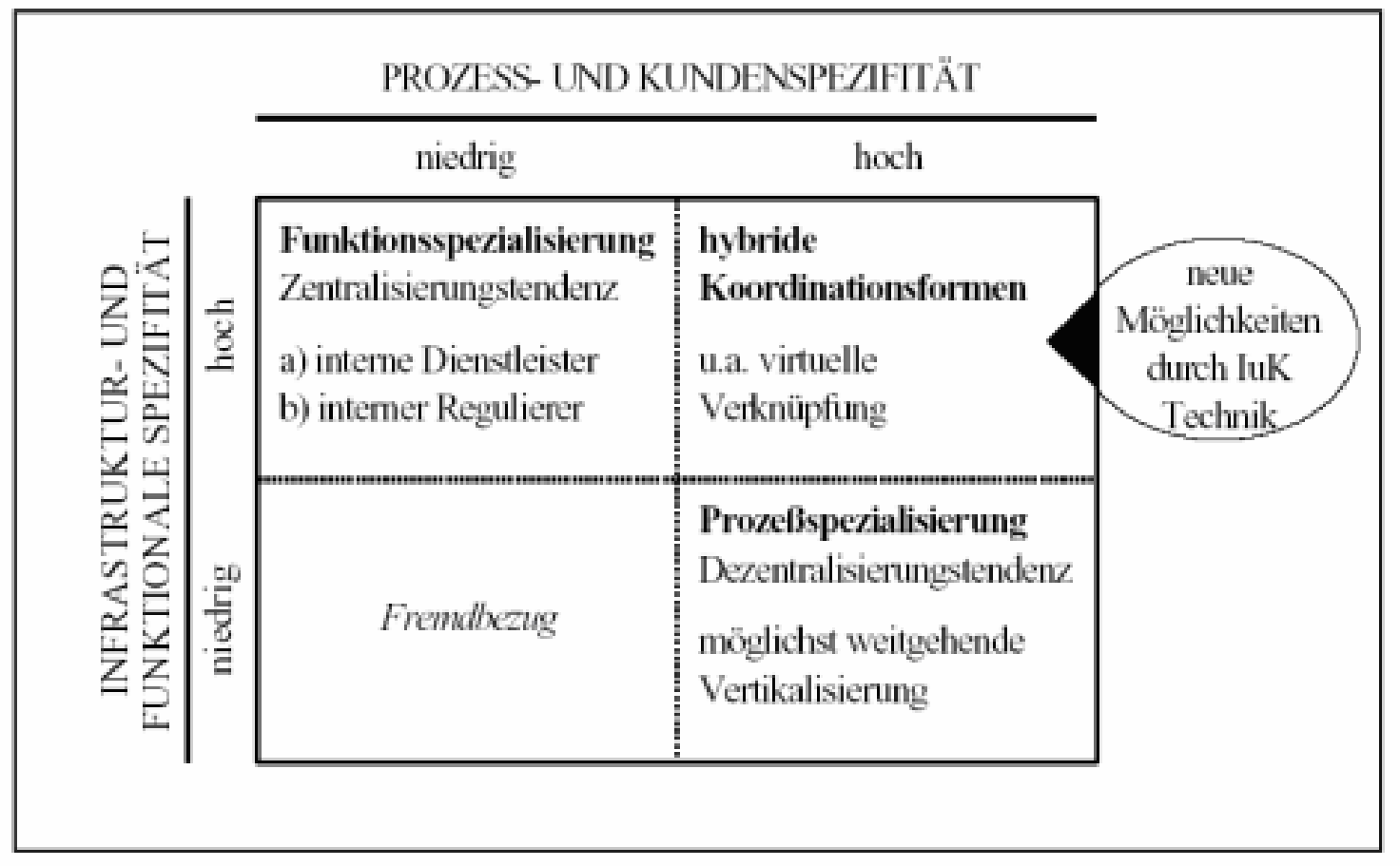

Abbildung 1: Einordnung hybrider Kooperationsformen [Picot01]

Auf dem Markt wird spontan über einzelne Wirtschaftssubjekte koordiniert, in einer Hierarchie durch feste Weisungsstrukturen. Hybride werden anhand folgender Merkmale identifiziert: „Koordination erfolgt über Kooperationsbeziehungen zwischen Wirtschaftssubjekten; dadurch kann eine prinzipielle Flexibilität in der Ausgestaltung der Kooperationsbeziehung erzielt werden. Hierbei kooperieren Unternehmen miteinander; Vereinbarungen relationaler Verträge bilden das Rahmenwerk.“ „Markt und Hierarchie sind hierbei als Endpunkte auf einem Kontinuum zu begreifen. Hybride Koordinationsformen sind z.B. strategische Allianzen, virtuelle Unternehmen und Joint Ventures.“

Hybride Wertschöpfungsketten sind demnach die Wertschöpfungsketten, die auf hybride Weise koordiniert werden. Für ihr Zustandekommen sind zumeist niedrigere Transaktionskosten gegenüber Markt und Hierarchie die Voraussetzung. Diese treten besonders bei hohen funktionalen, Prozess-, Kunden- und Infrastrukturspezifitäten auf (vgl. Abb. 1).

Unter Einbeziehung von verfügbaren Schlüsseltechnologien für hybride Kooperationsformen - vornehmlich aus dem Bereich der Informations- und Kommunikationstechnologien - und ihrer in der Entwicklung zu erwartenden Innovationen in einem globalen Markt stehen derzeit verschiedene Aspekte beim Wandel in Wertschöpfungsketten für Sachanlagen in der Diskussion:

- $\quad$ dieser Wandel ist an der Veränderung der Innovationstreiber erkennbar:

- Wettbewerb über Anschaffungskosten ersetzen durch Lebenszykluskosten

- Kompetenzzentrierung der Innovationsprozesse ersetzen durch multikompetente, inter-disziplinäre Innovationen

- Produktverbesserung ersetzen durch Nutzenverbesserung und Nutzungsergänzungen (produktbegleitende Dienste)

- Zentralisierung zwecks Rationalisierung ersetzen durch Dezentralisierung zwecks Servicesteigerung

- Nicht nur Produktionstechnologie, sondern auch Prozesswissen exportierbar machen (Produktstandards ergänzen durch Prozessstandards) 
Für die in diesem Beitrag behandelten Sachanlagen der Logistik erscheint der zuerst genannte, neue Innovationstreiber von besonderer Bedeutung zu sein:

Anlagenentwicklung, Nutzungsentwicklung, Prozessentwicklung

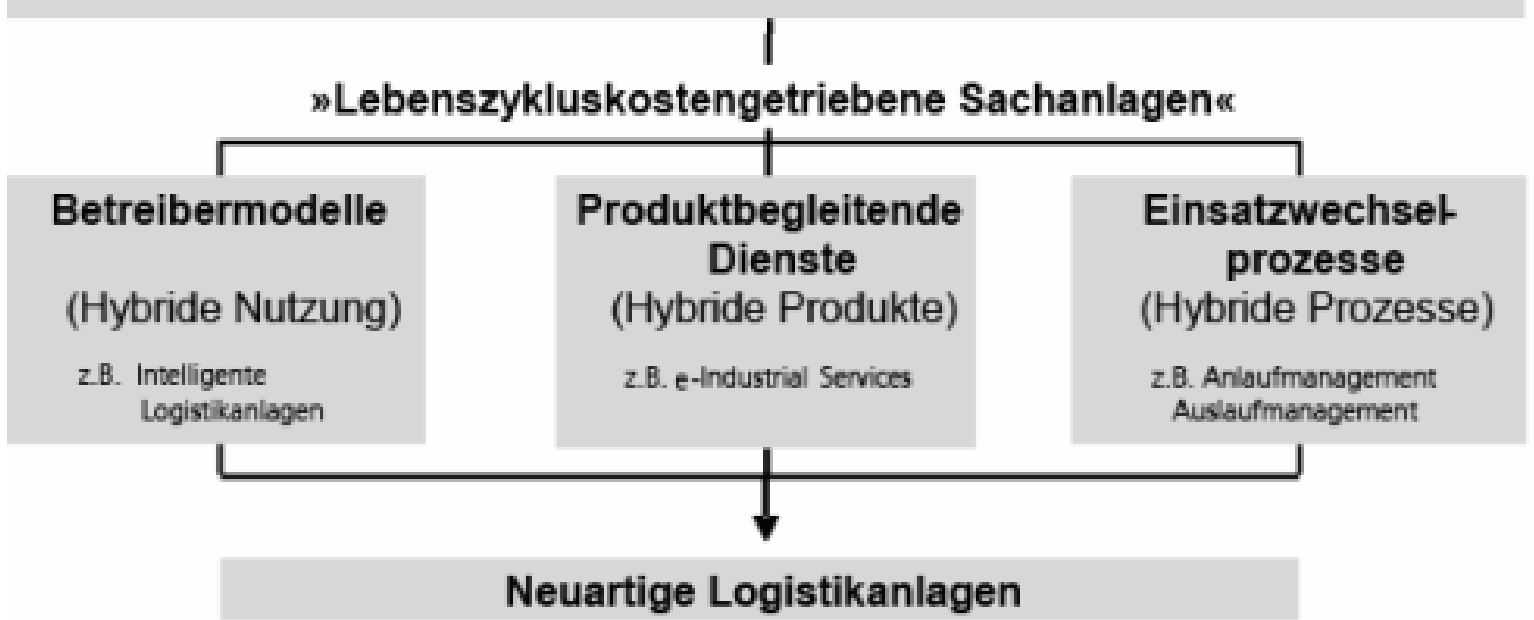

Abbildung 2: Kategorien von hybriden Koordinationsformen bei der Minimierung von Lebenszykluskosten für Logistikanlagen

In Abbildung 2 ist skizziert, in welchen Kategorien heute schon Veränderungen erkennbar sind:

Angesichts der immer kürzeren Nutzungsdauern einer Logistikanlage ist der Betreiber nicht mehr der Investor. Eine unveränderte Nutzung ist oft nur für max. drei Jahre garantiert. Anlagenlieferanten werden mit Forderungen nach Umnutzungsfähigkeiten bzw. -eigenschaften konfrontiert, die ihnen von den Eignern und den Betreibern diktiert werden. Im Extremfall wird der Anlagenlieferant zum Eigner - die Anlagen erhalten eine Wandlungsfähigkeit, die sowohl über modulare, konfigurierbare Technik, als auch über die Ertüchtigung der Steuerungs- und Integrations-Software erreicht wird

Anlagen müssen während ihrer Nutzung in optimalen Betriebsbereichen „gefahren“ werden. Die diversen Beeinflussungsmöglichkeiten (Leistungs- und Betriebskostenanpassungen) erfordern unterschiedliche Kompetenzen in den Bereichen der Systemplanung, der Software-Anpassung, der Wartung oder Instandhaltung, der Einsatzoptimierung von mobilen Betriebsmitteln, Integration neuer Technologien, Qualifizierung, Qualitätsmanagement, usw. Die Leistungen solcher Beiträge (Serviceleistungen) sind über Kennzahlen messbar. Sie sind zunehmend webfähig und flexibel abrufbar. Entsprechende Dienste sind im Zusammenhang mit der Anlage und ihrer Nutzung spezifiziert. Sie sind Bestandteil der Anlage (anlagenbegleitende Dienste Mehrwertdienste). Die Fraunhofer-Gesellschaft hat in einem großangelegten Eigenforschungsprojekt den Begriff „e-Industrial Services“ [Fraunhofer05] geprägt und deren Wiederverwendbarkeit und Übertragbarkeit untersucht.

Das Anlauf- bzw. Auslaufmanagement einer Anlagennutzung (Anlagenbetrieb) benötigt Vorgehensmodelle und Koordinierungsverfahren bzgl. vieler Ausstatter. Die entsprechenden Geschäftsprozesse erfordern heute noch zu viel Zeit. Die Aufgaben der Prozessverschmelzung und -auflösung in der Anbahnung und im Vollzug einer Anlagennutzung sind der Kategorie „Einsatzwechselprozesse“ von Anlagen zuzuordnen. Die hierbei zu beherrschenden Prozesse sind äußerst vielfältig und haben selten nur einen „Prozesseigner“, der mit eindeutigen Handlungskompetenzen ausgestattet ist.

Im Folgenden sollen nur die Potenziale produktbegleitender Dienstleistungen unter Punkt 2 untersucht werden, um den Wandel von hybriden Wertschöpfungsketten deutlich zu machen. Es muss konstatiert werden, dass noch viele gegenseitige Beeinflussungsarten der skizzierten Kategorien existieren. Die dargestellte Einordnung von Maßnahmen zur Minimierung der Lebenszykluskosten von Logistikanlagen stellen wir ausdrücklich zur Diskussion. 


\section{Produktbegleitende Dienste}

Durch die weitgehende Sättigung der weltweiten Märkte für Investitionsgüter wird sich der Wettbewerb zukünftig nicht mehr ausschließlich am technischen Entwicklungsstand eines Produktes orientieren. Der Problemlösungsbeitrag für den Kunden wird zu einem ergänzenden Erfolgsfaktor. Hersteller und Planer von Produktions- und Logistiksystemen werden zukünftig nur bestehen können, wenn sie ihr bisher isoliert entwickeltes, technisch orientiertes Produkt- oder Leistungsspektrum um produktbegleitende Dienstleistungen ergänzen [Bullinger99] und hybride Produkte anbieten.

Für Anlagenbetreiber sind diese (Mehrwert-) Dienstleistungen, die den wirtschaftlichen Nutzen einer Anlage über den gesamten Lebenszyklus signifikant erhöhen, von besonderer Bedeutung. Im Rahmen der strategischen Innovationsinitiative „e-Industrial Services“ [Fraunhofer05] werden von den führenden produktionstechnischen Instituten der Fraunhofer-Gesellschaft spezifische Mehrwertdienstleistungen zum „Verfügbarkeitsmanagement“, zur „adaptiven Personalqualifizierung“ und zur „Logistikplanung und -optimierung“ auf Basis komplexer internetbasierter anlagenspezifischer IT-Dienste entwickelt [Kuhn00].

Die Forschungs- und Entwicklungsschwerpunkte am Fraunhofer-Institut für Materialfluss und Logistik orientieren sich im Rahmen dieses Projektes am Lebenszyklus von Logistikanlagen. Eine Analyse zahlreicher durchgeführter Planungs- bzw. Umplanungsprojekte in der Produktion, der Beschaffung und der Distribution ergab, dass ein wachsender Bedarf an methodischer und werkzeugbezogener Unterstützung von ReengineeringAufgaben besteht. Die Konzeption und Entwicklung der Mehrwertdienstleistung der „Vorausschauenden Veränderungsplanung“ [Hellmann01] soll hier exemplarisch für viele weitere vorgestellt werden. Sie ordnet sich in die Vielzahl an Dienstleistungen im Lebenszyklus einer Anlage wie folgt ein:

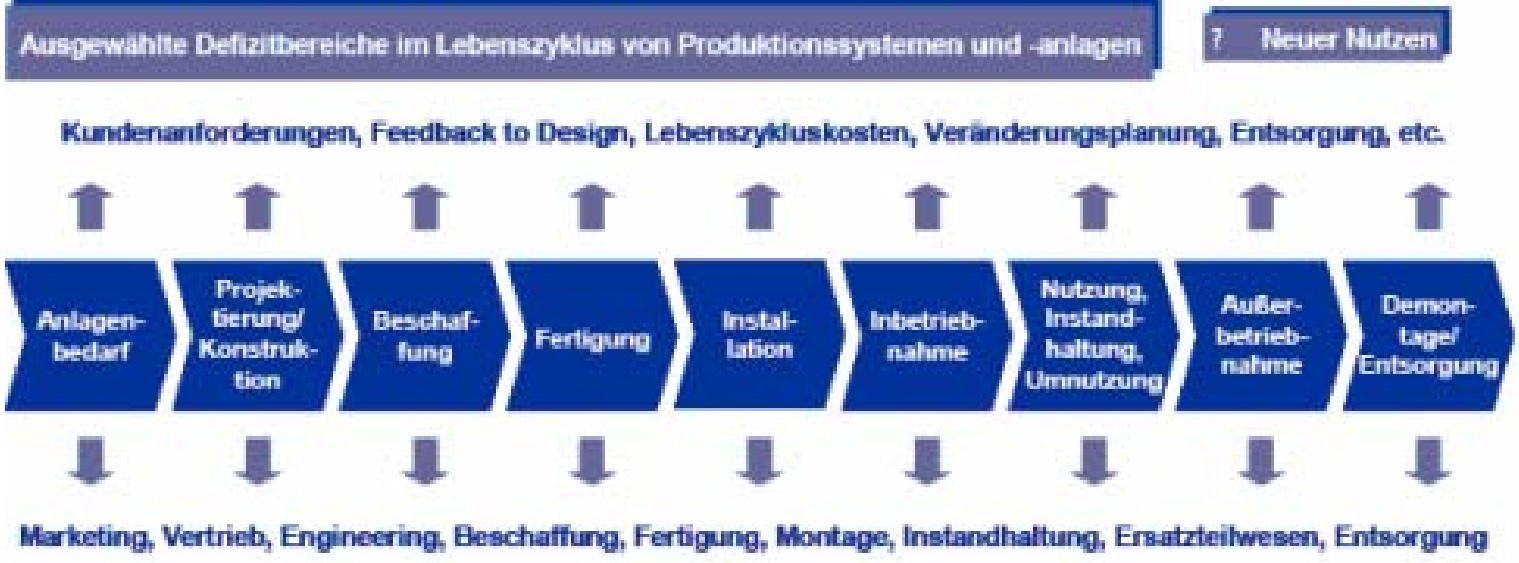

Benötigte Kompetenzen im Lebenszykdus von Produktionssystemen und -anlagen

Abbildung 3: Dienstleistungen im Lebenszyklus einer Anlage

Mehrwertdienste sind nur für konkrete und spezialisierte Anlagentypen und klar definierte Aufgaben darstellbar. Im Weiteren wird von dem Anlagentypus Lager, von weitgehend mechanisierten oder automatisierten Anlagen, die Rede sein.

\section{Ausgangssituation und Problemstellung}

In der Vergangenheit waren meist zu klein gewordene Lagerkapazitäten und die daraus folgenden notwendigen Erweiterungen eines Lagers Anlass für eine Überprüfungsplanung bestehender Anlagen. Heute bedingen zumeist Anforderungen einer verstärkten Kundenorientierung Kontrollen der installierten Anlagenkapazitäten und dynamiken. Gründe hierfür liegen in Verlagerungen von lager- zu kundenbezogener Fertigung bzw. in Auswirkungen der aufkommenden e-Commerce-Aktivitäten von Unternehmen mit zunehmender Atomisierung von Sendungen. Dies führt häufig zu einer Ausweitung der Artikelstrukturen, zu einer Erhöhung des Lagerplatzbedarfes bzw. steigenden dynamischen Anforderungen an Pick- oder Kommissionierleistungen. Diese stochastischen Eigenschaften des Marktes erfordern geänderte Methoden, um die Wirtschaftlichkeit des Lagerbetriebs zu sichern bzw. zu verbessern und setzen eine periodische Überprüfung des Lagerzustandes anhand von Prozessdaten, zugeschnittenen Parametern, Kennzahlen und Kennlinien voraus. 
Die vieldiskutierte Flexibilität der Anlagennutzung in der Produktion aufgrund häufig wechselnder Anforderungen gilt auch für Logistik- bzw. Lagersysteme. Um eine derartige flexible Anlagennutzung sicherstellen zu können, müssen folgende Eigenschaften installiert werden:

- $\quad$ kontinuierliche Überprüfung der installierten Leistung während des Betriebes,

- Ermittlung von Engpässen und Leistungsreserven während des Betriebes,

- $\quad$ kontinuierliche Überprüfung der Einhaltung optimaler, wirtschaftlicher Betriebsbereiche und

- Bereitstellung, Auswahl und optimierte Adaption alternativer Betriebsstrategien unter Berücksichtigung variabler Leistungsprofile (Systemvariationen).

Vorraussetzung für eine kontinuierliche Verbesserung des Lagerbetriebes ist die Verfügbarkeit einer aktuellen (Planungs-) Datenbasis. Im Falle einer konventionellen Überprüfungsplanung durch einen externen Dienstleister werden beim Lagerbetreiber notwendige Daten häufig noch manuell und aufwändig erhoben, an den Dienstleister versendet, dort manuell analysiert und individuell ausgewertet. Diese Prozesse sind zeit-, personalund kostenaufwändig. Auf Basis moderner Kommunikationsinfrastrukturen hat man die Möglichkeit, die beschriebenen Prozesse zur Erfassung, Aufbereitung und Bewertung der Planungsdatenbasis zu vereinfachen, zu automatisieren und zu beschleunigen.

Der Einsatz einer Simulation als ein Werkzeug zur Absicherung komplexer Planungen ist heutzutage nicht nur in der Wissenschaft, sondern auch im industriellen Umfeld Stand der Technik. In der Logistiksystemplanung wird die Methodik der Materialflusssimulation seit Mitte der 80er Jahre effizient eingesetzt und es existiert eine Vielzahl von kommerziellen Simulationstools (z.B. eMPlant, TaylorED, Automod, Simpro). Der weiterführende Einsatz der zur Planungsabsicherung erstellten Simulationsmodelle zur betriebsbegleitenden Bewertung von Logistiksystemen ist jedoch sehr selten; entsprechende Dienste sind nicht etabliert.

Vor diesem Hintergrund steht die Konzeption und Entwicklung des internetgestützten Mehrwertdienstes der „Vorausschauenden Veränderungsplanung“.

\subsection{Lösungsansätze zur „betriebsbegleitenden simulationsgestützten Bewertung von Logistiksystemen“}

Voraussetzung für eine kostengünstige Durchführung eines Mehrwertdienstes zum betriebsbegleitenden Monitoring von Logistiksystemen ist eine bereits initialisierte Projektinfrastruktur, die idealer Weise im Rahmen der Anlagenplanung beispielsweise auf Basis des Mehrwertdienstes „Angebotsmanagement“ aufgebaut wurde. Sie ermöglicht die Kontrolle und Steuerung des Zugriffes auf Daten und Dokumente, idealer Weise über den gesamten Lebenszyklus einer Anlage. Ferner beinhaltet sie u.a. Anlagenbeschreibungen in Form von Simulations- und Referenzprozessmodellen sowie eine aktualisierte Datenbasis mit Stamm- und Prozessdaten, die in der Betriebsphase periodisch aktualisiert wird. Das systembegleitende Datenmanagement bildet die Grundlage aller Mehrwertdienste für Logistiksysteme des Fraunhofer IML und verwaltet sowohl die Daten aus Inhouse-Systemen der Logistikanlage (z.B. Auftragsdaten) als auch die Daten der Anlagenplanung (z.B. Layoutparameter) und der Optimierung/Simulation (z.B. Steuerungsstrategien, Auftragsreihenfolgen, Kennlinien) - also auch der „Vorausschauenden Veränderungsplanung“.

Existiert eine solche digitale Anlagenbeschreibung bzw. -modellierung nicht, so ist eine wirtschaftliche Realisierung des angesprochenen Dienstes kaum möglich. Diese Aussage belegt die Tatsache, dass Mehrwertdienste über den Gesamtlebenszyklus einer Anlage geplant und definiert sein müssen - vielleicht ein Grund dafür, dass Lösungen für Mehrwertdienste im Sinne dieses Anspruchs nicht oder höchst selten existieren. 


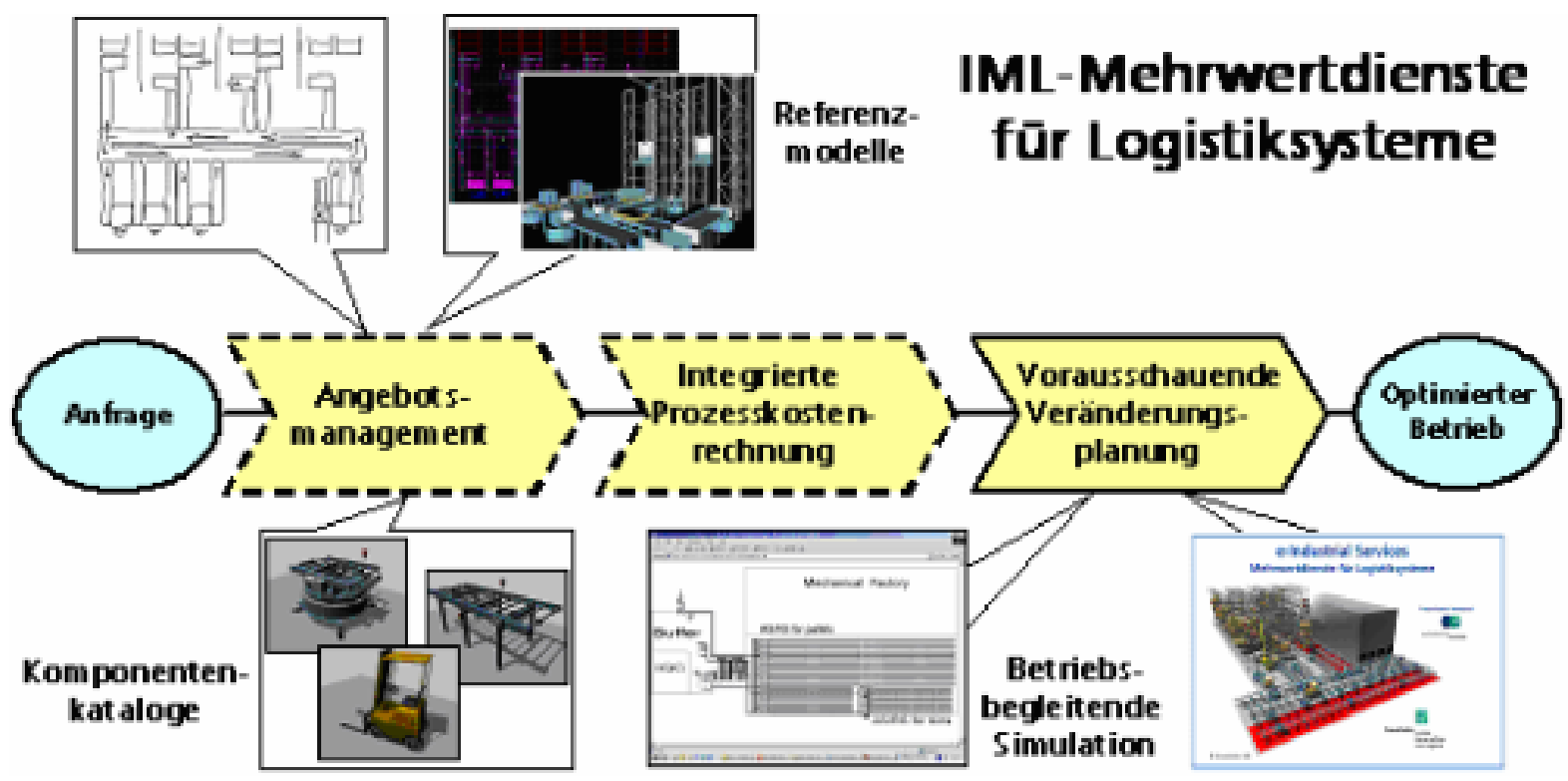

Abbildung 4: Übersicht der „e-Industrial Services“ des Fraunhofer IML

Das Prozesskettendiagramm in Abbildung 5 zeigt den funktionalen Ablauf des Mehrwertdienstes „Vorausschauende Veränderungsplanung“, der in den folgenden Abschnitten detailliert erläutert wird. Grundsätzlich werden zwei Hauptprozesse unterschieden. Der betriebsbegleitende Prozess „periodischer Check“ hat die Zielsetzung des Monitoring, der Analyse und Bewertung einer bestehenden Logistikanlage. Der Prozess „Veränderungsplanung durchführen“ nutzt das bestehende Datenmanagement, diverse Komponentenkataloge, Simulations- und 3D-Modelle, sowie Umplanungs- bzw. Erweiterungplanungserfahrungen der Anlagenlieferanten, um eine Logistikanlage schnell und effizient umzugestalten. Das Simulationsexperiment bewertet die erreichten Verbesserungspotenziale im Vergleich zum Monitoringergebnis der laufenden Anlage (Validierung des Simulationsmodells) mit den Ist- und Sollsystemlasten.

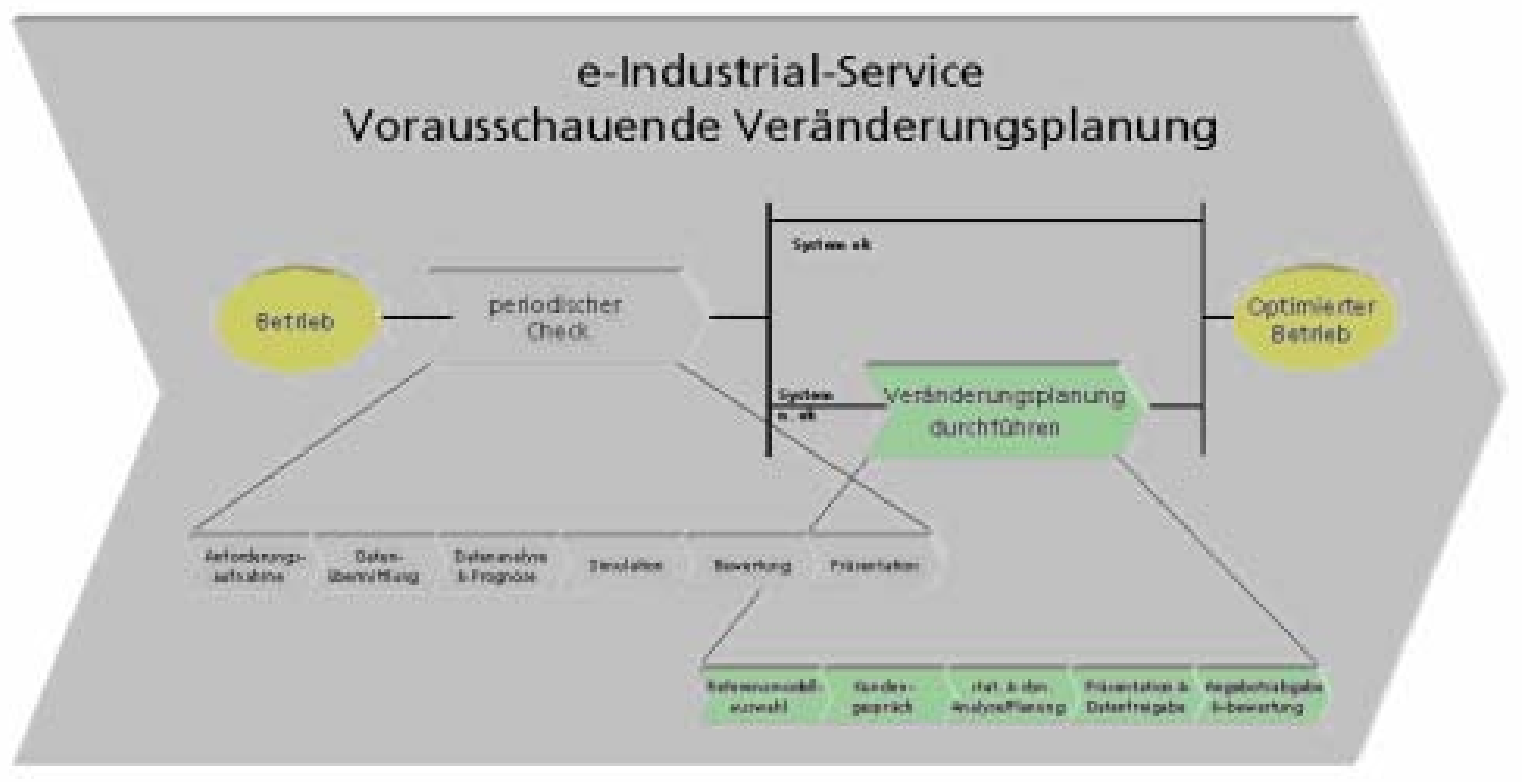

Abbildung 5: Prozesskette des simulationsbasierten „e-Industrial Service“ der „Vorausschauenden Veränderungsplanung“

\subsubsection{Prozess „Periodischer Check“}

Die Diensterbringung wird durch einen Kundenimpuls ausgelöst, der entweder in periodischer oder einmaliger Form erfolgen kann. Ein periodischer Kundenimpuls wird z.B. durch einen Rahmenvertrag für eine regelmäßig durchzuführende Überprüfung der Anlagenkapazitäten und -strukturen ausgelöst. Ändert ein Kunde seine Unternehmensziele oder -strategien, kann er eine einmalige kapazitive Überprüfung seiner Ressourcen initiieren. 
Bei einem derartigen außerperiodischen Kundenimpuls werden die neu abzubildenden normativen Randbedingungen neuer Unternehmensziele, Leistungs- oder Betriebsstrategien aufgenommen. Hierbei helfen Checklisten, die ein systematisiertes und strukturiertes Vorgehen bewirken. Anschließend werden die im Steuerungssystem der Logistikanlage vorliegenden Stamm- und Prozessdaten an den Diensterbringer übermittelt. Ebenfalls sind die aufbereiteten Anforderungsinformationen des Anlagennutzers bereitzustellen (Prozess „Datenübermittlung“). Nach Abschluss dieses Prozesses werden beim Diensterbringer die in der Betriebsphase gesammelten Daten gefiltert und aggregiert (Prozess „Datenaufbereitung“). Somit steht für die nachfolgenden Prozesse eine gefilterte und aufbereitete Datenbasis zur weiteren Untersuchung zur Verfügung. Im Rahmen einer statischen Analyse werden diese Daten zunächst selektiert und zu Kennzahlen und Kennlinien verdichtet. Um eine vorausschauende Analyse und Simulation der Logistikanlage durchführen zu können, werden die verdichteten Lastdaten anschließend im Sinne einer Verlängerung der Ist-Situation in die nähere Zukunft mit Hilfe von adäquaten Prognoseverfahren aufbereitet (Prozess „Datenanalyse \& Prognose“).

Die aufbereiteten Systemlastdaten werden dann mit Hilfe von Simulationsmodellen dynamisch analysiert (Prozess „Simulation“). Anschließend erfolgt eine systembezogene Bewertung der Simulationsergebnisse, in die auch normative Informationen (Unternehmensziele, Strategien, ...) bezüglich des Systemnutzers einfließen können. Eine monetäre Bewertung von verschiedenen Systemlastvarianten kann unter anderem durch das Modul der ressourcenorientierten Prozesskostenrechnung erfolgen (Prozess „Bewertung“) [Fuchs04]. Der Prozess der „Präsentation“ umfasst die Darstellung der Ergebnisse in der vom Anlagennutzer gewünschten Form. Ist keine relevante Diskrepanz zu erkennen - d.h. die Anlage wird in ihrem vorgesehenen Betriebsbereich genutzt - wird lediglich eine informatorische Meldung an den Anlagennutzer abgesetzt. Ist jedoch eine zukünftig relevante und kapazitive Unter- oder Überdeckung erkennbar („System nicht ok“), wird der Prozess der strukturellen und problemorientierten "Veränderungsplanung" angestoßen.

\subsubsection{Prozess „Veränderungsplanung durchführen“}

Dieser Prozess bietet die Möglichkeit, Umplanungen bzw. Erweiterungen der Logistikanlage schnell und effizient zu gestalten. Diese Planung wird sowohl durch die bestehende Projektinfrastruktur in Form von Simulations-, 3D-Modellen („Walk-through“), einer aktualisierten Datenbasis mit Stamm- und Prozessdaten, als auch durch das System- und Planungs-Know-How des Diensterbringers unterstützt. Zum anderen vereinfacht und beschleunigt eine bestehende Kommunikationsstruktur zwischen den Beteiligten (Anlagennutzer, Anlagenhersteller und Diensterbringer) die Projektabwicklung erheblich.

Ausgangspunkt einer Veränderungsplanung ist eine erkannte Diskrepanz zwischen Anlagenkapazitäten bzw. strukturen und Kunden- bzw. Leistungsbedarfen. Ziel ist es, Umplanungen der Logistikanlage möglichst schnell und aufwandsarm durchzuführen. Hierzu werden die direkt bestehenden Kommunikationsstrukturen zwischen Anlagennutzer, -hersteller und Diensterbringer über die Plattform genutzt.

Auf Basis der bereits bestehenden Planungen und der erkannten Änderungsbedarfe können prinzipielle Planungsvarianten bereits im Vorfeld in Form von Referenzmodellen (Simulationsmodell, 3D-Modell für ein "Walk-through"-Erlebnis) ausgewählt werden (Prozess „Referenzmodellauswahl“). Unter Verwendung dieser Referenzmodelle als Kommunikationsbasis werden im Dialog mit dem Anlagennutzer konkrete Lösungmöglichkeiten erarbeitet. Insbesondere das 3D-Modell schafft durch ein "Walk-through"-Erlebnis ein verbessertes gemeinsames Verständnis des Planungsgegenstandes (Prozess „Kundengespräch“). Ziel des Prozesses „statische \& dynamische Analyse und Planung“ ist die Erstellung der technischen Ausschreibungsunterlagen für die Änderungsplanung. Hierbei können elektronische Komponentenkataloge zum Einsatz kommen, die in Zusammenarbeit mit dem Anlagenhersteller gepflegt werden und die gängigen Verfahren der statischen und dynamischen Analyse sowie der Planung unterstützen. Zur „Präsentation und Abnahme“ werden die erarbeiteten Ausschreibungsunterlagen präsentiert und vom Anlagennutzer freigegeben. Bei Nichtabnahme erfolgt eine Rückverweisung an die „statische und dynamische Analyse / Planung“. Die hierdurch entstehende Iteration zwischen Planer und Anlagennutzer kann mehrmals durchlaufen werden. Ergebnis dieses Prozesses sind die vom Anlagennutzer abgesegneten, technischen Dokumente der Ausschreibung, die für Anlagenhersteller zur Angebotserstellung freigegeben werden.

Auf Basis der freigegebenen Ausschreibungsdokumente des Anlagennutzers können partizipierende Anlagenlieferanten Angebote mit Zugriff über die Plattform erstellen. Die abgegebenen Angebote werden vom Diensterbringer geprüft und die Bewertung dem Anlagennutzer vorgelegt. Bei Annahme des Angebotes durch den Anlagennutzer wird der Anlagenhersteller formal beauftragt und im Rahmen der Realisierung die Projektinfrastruktur um diesen Auftrag erweitert [Jessen01], [Hellmann03]. 


\subsubsection{Technische Infrastrukturkomponenten}

Die zur Entwicklung von elektronisch erbrachten Dienstleistungen notwendigen Basistechnologien sind seit längerer Zeit vorhanden und besitzen in den IT-Abteilungen bereits eine hohe Akzeptanz. Viele Funktionen sind bislang jedoch nur als Einzellösungen auf Basis von Internet-Technologien verfügbar. Häufig ist es notwendig, diese Bausteine bei Applikationsentwicklungen neu $\mathrm{zu}$ implementieren oder zu integrieren. Es ist daher naheliegend, die benötigten Infrastrukturkomponenten auf einer Internetplattform zu bündeln und den Applikationsentwicklern als Tool-Baukasten anzubieten. Vor diesem Hintergrund wird im Rahmen des zugrunde liegenden Forschungsprojektes eine „Service-Plattform“ zur vereinfachten Implementierung und Erbringung von e-Industrial Services entwickelt [Hohwieler01].
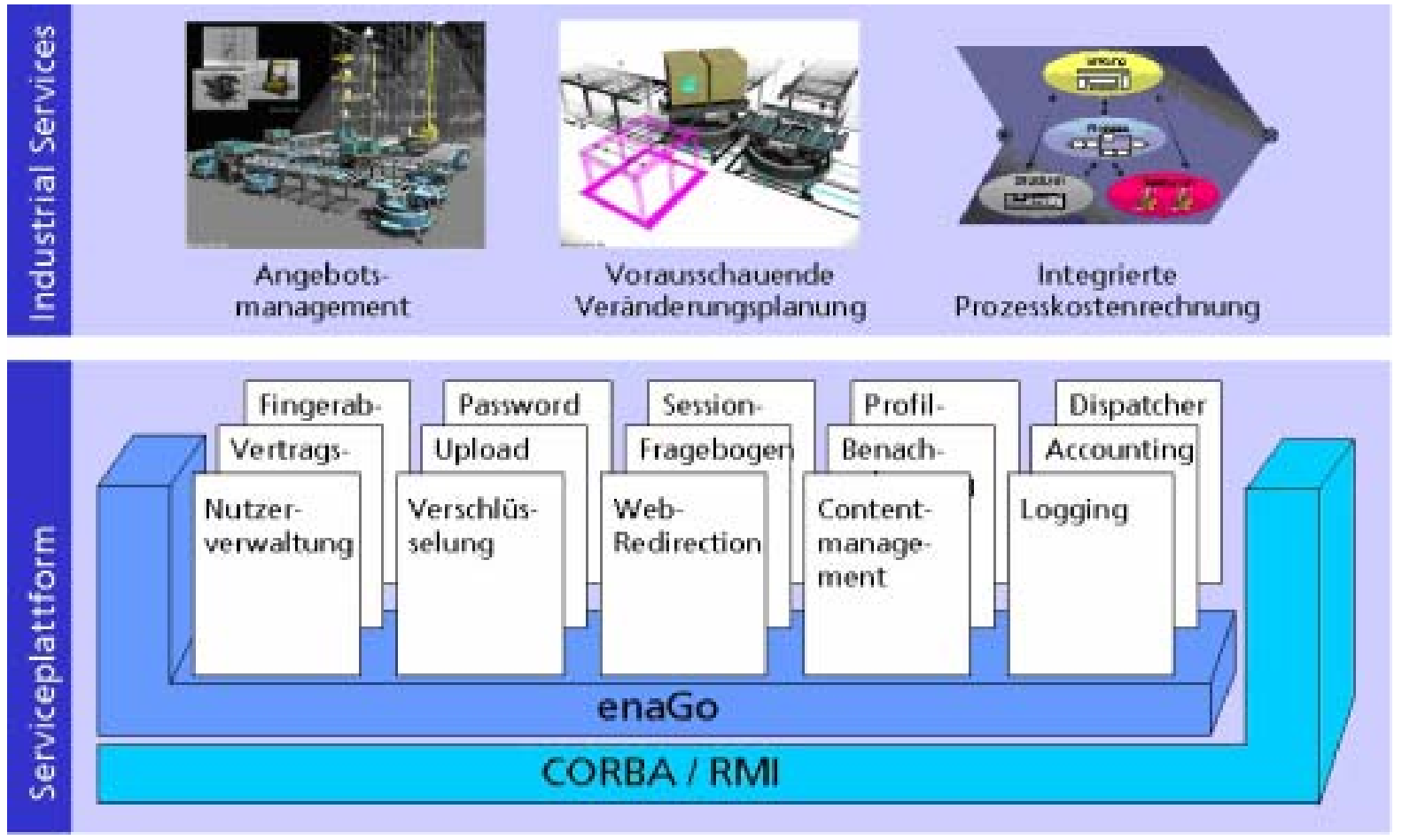

Abbildung 6: Komponenten der Mehrwertdienste.,,Service Plattform“

Sie basiert maßgeblich auf zwei Softwarekomponenten: Zum einen der Plattform-Software „enaGO“ des Fraunhofer-Instituts für offene Kommunikationssysteme (Fokus) in Berlin und zum anderen dem ContentManagement-System (CMS) „WebGenesis“ des Fraunhofer-Institut für Informations- und Datenverarbeitung (IITB) in Karlsruhe. Der Kern der Plattform basiert aus Gründen der Ausführungsgeschwindigkeit auf der Programmiersprache C++. Zur Realisierung einzelner Dienste auf der „Service Plattform“ kommt aufgrund der hohen Entwicklungs-Performance die Progammiersprache Java zum Einsatz.

Die Plattformsoftware „enaGO“ bietet Unterstützung bei Entwicklung und Betrieb von.internetbasierten Dienstleistungen und übernimmt Standardaufgaben, wie Authentifizierung und Autorisierung. Das bereitgestellte Framework für Java und $\mathrm{C}++$ erlaubt dem Dienstentwickler auf Nutzerinformationen zuzugreifen und dienstspezifische Informationen in einem Nutzerprofil zu speichern. So sind umfassende Möglichkeiten der Personalisierung von Diensten gegeben. Das Dienstentwicklungsmodell basiert auf der konsequenten Trennung von Nutzerschnittstelle und Dienstlogik in jeweils getrennten Anwendungen. Die flexible und umfangreiche Nutzer- und Sitzungsverwaltung ist eine Kernkomponente der Plattform und ermöglicht komplexe Rollenmodelle.

„WebGenesis“ ist ein modulares CMS sowohl für den Aufbau von Web-Präsentationen im Internet-Bereich als auch als Wissensserver für Unternehmen im Intranet-Bereich. Die modulare Struktur des Systems erlaubt, neben der Verwaltung von Web-Inhalten, den Aufbau von Internet-Anwendungen, die über die reine Informationspräsentation hinausgehen. Als ein Beispiel hierfür wird nachfolgend der Demonstrator der eIndustrial Service der „Vorausschauenden Veränderungsplanung“ vorgestellt.

\subsubsection{Fallbeispiel: Demonstrator der „Vorausschauenden Veränderungs-planung“}

Im Rahmen des Verbundforschungsprojektes wurde der Mehrwertdienst in Zusammenarbeit mit einem Industriepartner prototypisch auf Basis der beschriebenen Service-Plattform realisiert. 
Am Beispiel eines Hochregallagers für Paletten und Behälter mit Wareneingang, Konturencheck, Ein/Auslagerfördertechnik sowie Lager und Kommissionierbereich wurden in einem Demonstrator die typischen Komponenten zur betriebsbegleitenden simulationsgestützten Bewertung eines Logistiksystems abgebildet [Bullinger99].

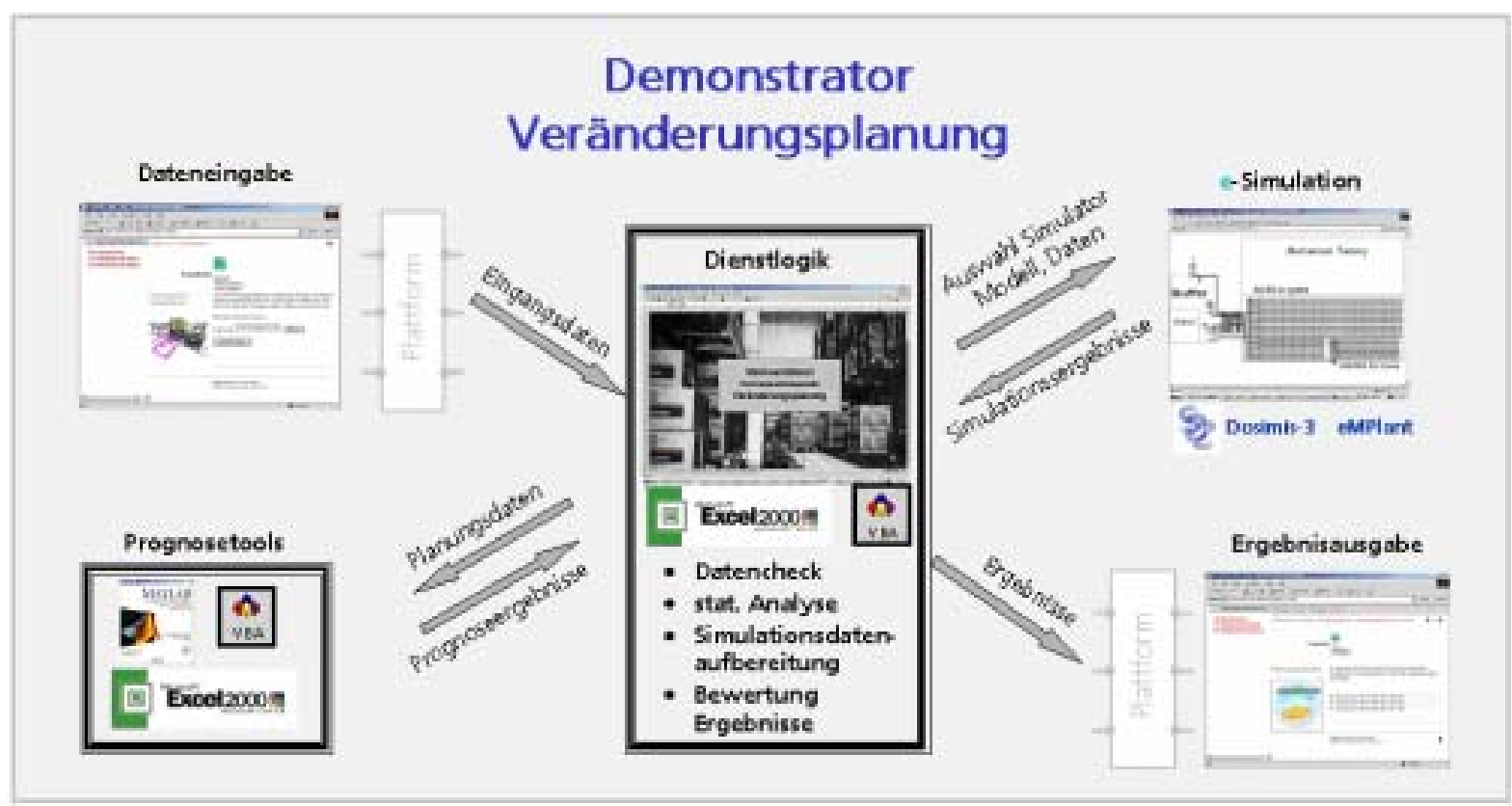

Abbildung 7: Technischer Aufbau des Demonstrators des e-Industrial Service der „Vorausschauenden Veränderungsplanung“

Die Kernkomponente des Demonstrators bildet ein Simulationsmodell der betrachteten Anlage. Es wurde im Rahmen der Systemplanung so konzipiert, dass wichtige Parameter der Simulation (z.B. Auftragsdaten, Taktzeiten, Kapazitäten, etc.) getrennt vom Simulationsmodell gespeichert sind. Dies ermöglicht dem Anwender eine Nutzung des späteren Mehrwertdienstes ohne tiefgehende Kenntnisse des eingesetzten Simulationswerkzeuges. Es können Szenarien definiert werden, in denen die Möglichkeit der Variation zum einen von strukturellen Parametern (z.B. Prognosezeitraum, Anzahl Kommissionierplätze) oder zum anderen von Eingangsdaten (z.B. Auftragsdaten) besteht. Diese Daten werden vom Dienstnutzer entweder über einen Upload-Service manuell zum Service-Provider übermittelt oder, im Falle einer automatisierten Überwachung, einer Logistikanlage in zyklischen Abständen automatisiert über einen FTP-Server bereitgestellt. Beim ServiceProvider werden die eingegangenen Daten anwenderspezifisch im integrierten CMS gespeichert. Anschließend startet in der Komponente Dienstlogik ein Konsistenz-Check einschließlich einer statischen Analyse und die Übergabe der aufbereiteten Daten an die Prognosekomponente.

Für die Prognose der zukünftigen Systemlasten wurden im e-Service verschiedene Prognosemethoden und werkzeuge (Matlab, Excel) eingebunden, die in Abhängigkeit von den Inputdaten ausgewählt werden können. Es stehen sowohl modellbasierte Verfahren als auch numerische Verfahren (z.B. Trendanalyse mit saisonalem Pattern) zur Verfügung. Die Auswahl des jeweils passenden Verfahrens wird im Rahmen der Systemplanung analysiert und für den Anwender vorparametriert. Nach Durchlauf der Prognosekomponente werden die Eingangsdaten an die Simulationskomponente übergeben und diese gestartet.

In die Simulationskomponente des Mehrwertdienstes können prinzipiell beliebige diskrete Materialflusssimulatoren eingebunden werden. Zurzeit sind Schnittstellen zu den Werkzeugen eMPlant (Tecnomatix Technologies Ltd.) und DOSIMIS3 (SDZ GmbH) in Verbindung mit CASUS (Fraunhofer IML) implementiert.

Nach Beendung und Auswertung der Simulation, erhält der Anwender die Ergebnisse in Form von Tabellen und Grafiken über die Serviceplattform im CMS in einem nur für ihn zugänglichen Bereich zur Verfügung gestellt. Die Form der Auswertungen wird jeweils im Rahmen der Dienstparametrisierung kundenspezifisch ausgewählt. Falls die Simulation zukünftige Systemengpässe aufzeigt, erfolgt eine aktive Benachrichtigung der Anlagennutzer und der automatisierte Teil des Mehrwertdienstes im Demonstrator ist abgeschlossen. 


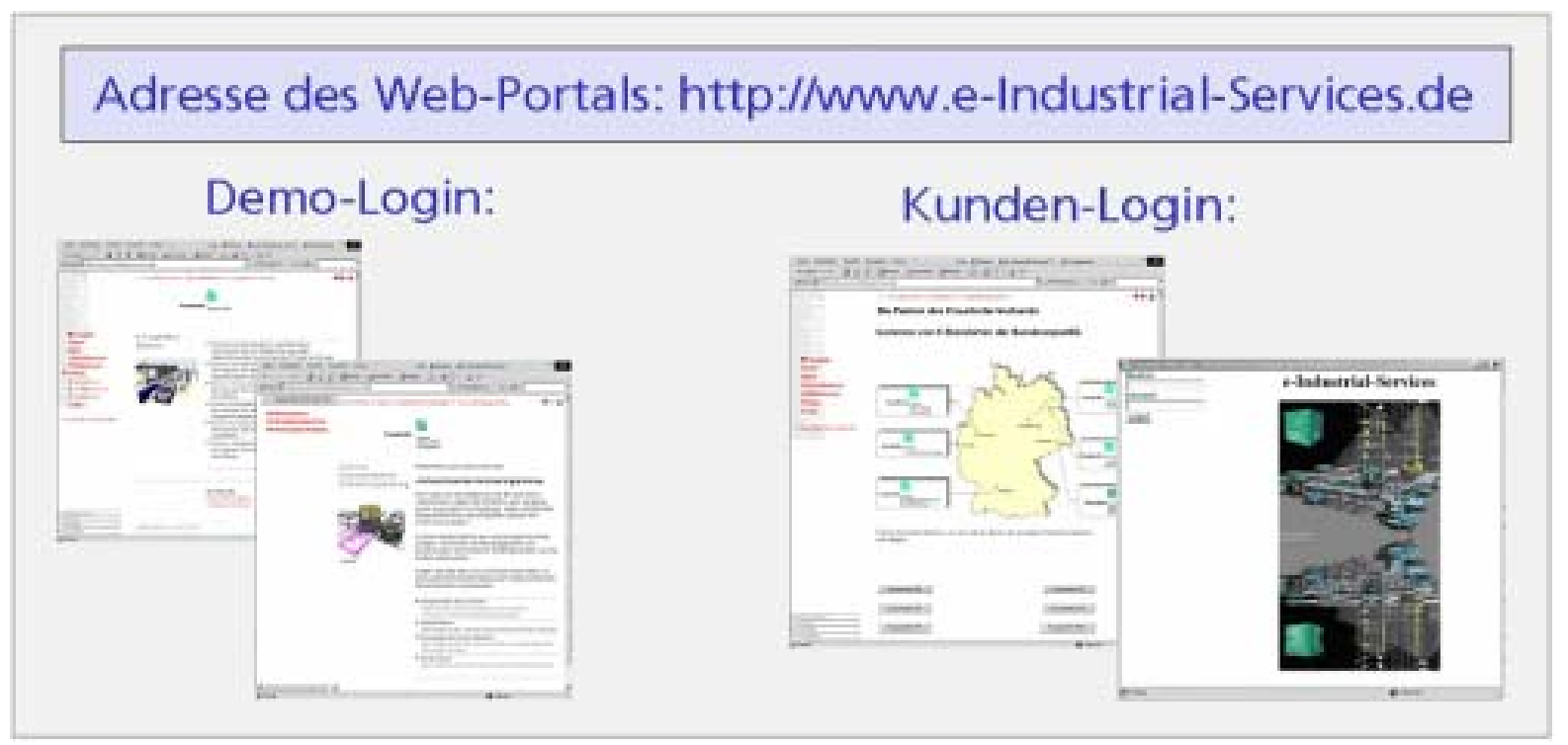

Abbildung 8: Web-Portal des Forschungsprojektes „e-Industrial Services“ mit Zugang zu den verschiedenen Demonstratoren und Kunden-Login

\section{Zusammenfassung}

Aus dem großen aufgespannten Forschungs- und Entwicklungsfeld der hybriden Wertschöpfungsketten wurden in diesem Artikel die Sachanlagen der Logistik als Repräsentant lebenszyklusgetriebener Produkte herausgegriffen und die besondere Bedeutung der Innovationstreiber „Betreibermodelle“, „Produktbegleitende Dienste“ und „Einsatzwechselprozesse“ skizziert. Am Beispiel der „Produktbegleitenden Dienste“ wurde aufgezeigt, welche Wissens- und Kompetenzbereiche mit der Anlage zu Mehrwertdiensten verbunden werden können. Am Beispiel einer prototypischen Realisierung für einen dieser Dienste, der vorausschauenden Veränderungsplanung zur modellgestützten Systemwartung und -anpassung, wurde aufgezeigt, wie neue Technologien für den Mehrwertdienst einer Logistikanlage genutzt und in die Anlage integriert werden können. Diese prototypische Lösung zeigt die Potenziale einer großen und lohnenden Forschungs- und Entwicklungsaufgabe auf.

\section{Literaturverzeichnis}

[Bullinger99]

[Duden04]

[Fraunhofer05]

[Fuchs04]

[Hellmann01]

[Hellmann03]

[Hohwieler01]

[Jessen01]

[Kuhn00]

[Picot01]
Bullinger, Hans-Jörg: Dienstleistungen : Innovation für Wachstum und Beschäftigung. Herausforderung des internationalen Wettbewerbs, Wiesbaden: Gabler, 1999

Duden : Deutsche Rechtschreibung. 23. Auflage. Mannheim: Dudenverlag, 2004

Fraunhofer Verbundprojekt e-Industrial Services [http://www.e-industrialservices.de], Datum des Zugriffs 25.10.2005

Fuchs, Frank : Entwicklung eines Werkzeugs zur ressourcenorientierten Prozesskostenrechnung für die Logistik. Dissertation, Dortmund: Praxiswissen, 2004 Hellmann, Andreas; Fuchs, Frank: Mehrwertdienste für Sachanlagen der Logistik. In: Jahrbuch der Logistik 2001, Düsseldorf: Handelsblatt-Verlag, 2001 Hellmann, A.; Jessen, U.; Wenzel, S. : e-Services - A Part of the "Digital Factory”. In: Bley, H. (Hrsg.): Proceedings of the 36th CIRP International Seminar on Manufacturing Systems, Saarbrücken 2003, S. 199-203

Hohwieler, Eckhard, Berger, Ralf: Serviceplattform für industrielle Dienstleistungen im Internet. In: Futur Magazin (2001) 1, S. 6-7

Jessen, Ulrich, Wenzel, Sigrid: Elektronische Kataloge unterstützen den Fabrikplanungsprozess. In: Jahrbuch der Logistik 2001, Düsseldorf: HandelsblattVerlag, 2001

Kuhn, Axel: Mehrwertdienste in der Logistik. In: Logistik Sonderausgabe Distribution 31 (2000) 10, S. 17-19.

Picot, Arnold; Reichwald, Ralf; Wiegand, Rolf T.: Die grenzenlose Unternehmung, 4. Auflage. Wiesbaden: Gabler, 2001 